\title{
Transcript-specific regulation in T-cells in multiple sclerosis susceptibility
}

\author{
Lies Van Horebeek ${ }^{1,2} \cdot$ An Goris $\mathbb{D}^{1,2}$
}

Received: 11 February 2020 / Accepted: 12 March 2020 / Published online: 2 April 2020

(c) European Society of Human Genetics 2020

Multiple sclerosis (MS) is an autoimmune disease of the central nervous system. Association with the Human Leukocyte Antigen region has long been known, and over the past decade genome-wide association studies (GWASs) have identified many single nucleotide variants (SNVs) associated with disease. As typical for complex diseases, the vast majority of these are common (allele frequency $>5 \%$ ), have a modest effect on susceptibility (odds ratio $<1.2$ ) and are noncoding. MS risk variants are enriched in regulatory sites controlling gene expression in immune cells. Hence, GWAS points to a predominant role of the immune system in MS pathogenesis.

Unraveling the mechanism of action of risk variants is a current key challenge that cannot be addressed by GWAS only. Detailed mechanistic studies are limited to a handful of variants. In this issue, Ban et al. from Cambridge University adopt a well-designed systematic approach screening $110 \mathrm{MS}$ risk loci for allele-specific expression (ASE) [1]. This work forms an extensive resource translating MS genetic risk variants to prioritized candidate genes, and highlights risk variants affecting transcript-specific regulation. Results from this elegant application of ASE in MS by Ban et al. [1] are an excellent illustration of the emerging role of genetic variants modulating alternative splicing and transcript-specific expression in susceptibility to complex disease [2, 3], besides their already well-known role in Mendelian disease.

Expression or splicing quantitative trait loci (eQTL or sQTL) can be detected by differences in expression levels across a large sample of individuals with different genotypes. ASE detection is a robust and sensitive alternative

An Goris

an.goris@kuleuven.be

1 KU Leuven - Department of Neurosciences, Laboratory for Neuroimmunology, 3000 Leuven, Belgium

2 Leuven Brain Institute, 3000 Leuven, Belgium approach to identify these regulatory SNVs through an imbalance in expression of both alleles within heterozygote individuals. Despite technical constraints enabling ASE detection, Ban et al. could interrogate 60 out of 110 MSassociated loci using 200 coding SNVs in linkage disequilibrium with the MS-associated variants from 112 flanking genes. At a Bonferroni-corrected significance threshold $\left(p<5 \times 10^{-5}\right)$, half of the investigated loci (29 out of 60) show significant ASE (56 coding variants implicating 43 flanking genes) corresponding to an average 50\% increase in reads containing one allele (risk/protective) compared with the other allele. Many of the MS-associated SNVs alter the balance of alternate transcripts, although the targeted sequencing approach did not allow to formally distinguish eQTLs from sQTLs. Furthermore, 17 out of 56 significant ASE signals implicate a noncoding transcript. Of note, sQTLs as a group are enriched for association with MS, even when compared with eQTLs [3].

Different transcripts can have distinct regulatory properties and can be translated into stable protein isoforms with divergent structures and functions. Ban et al. detect ASE for MS risk variants known for transcript-specific regulation. TNFRSF1A, IL7R, and SP140 risk variants are compatible with increased exon exclusion, leading to more soluble decoy receptor lacking the transmembrane domain (TNFRSF1A, IL7R) or more protein with an in-frame 26 amino acid deletion (SP140). A CD40 variant shared between multiple autoimmune diseases influences alternative splicing, a mechanism part of cell activation and CD40 upregulation. The most prominent new finding in this study concerns an MS-associated variant on chromosome 20 with three flanking genes investigated. In both the screening (211 individuals) and replication cohort (189 individuals), ASE implicates the MS risk variant in transcript-specific regulation of one gene, LIME1 (Fig. 1). A strength of the study is the inclusion of both cases and controls, and the ASE effect appears more prominent in MS patients than healthy controls. It remains to be determined whether and how different LIME1 transcripts affect the 
LIME1

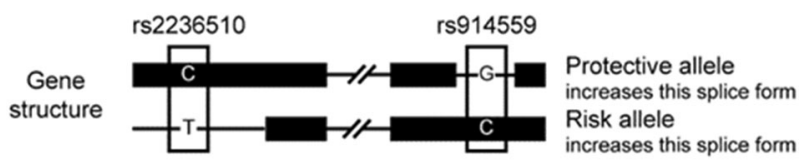

Reads
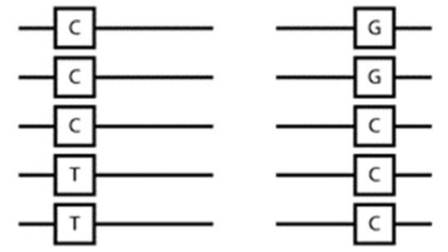

Fig. 1 Detection of allele-specific expression of LIME1. Gene structure with exons and introns for part of LIME1 (not on scale) and two coding variants in linkage disequilibrium with the MS-associated variant rs2256814. MS risk allele is associated with opposite effects for different transcripts, i.e., with reduced expression of transcripts containing rs2236510 and increased expression of transcripts containing rs914559.

known involvement of this gene in T-cell receptor signaling.

Once a disease variant is associated with ASE and/or an eQTL, it remains to be established whether both the disease and the expression association are driven by the same underlying effect. This is likely the case for the chromosome 12 region, where one of five flanking genes, METTL21B, displays strongest ASE in T-cells with the risk variant reducing expression. Fine-mapping approaches indicate a shared genetic effect driving expression and disease association [4]. In regions of high linkage disequilibrium, such as the chromosome 17 region, or with multiple regulatory variants, it remains more difficult to pinpoint a particular candidate gene and to demonstrate a shared underlying effect. Here, additional research is required.

The recently updated list of MS loci implicates not only T-cells, the focus of this study, but also B-cells and innate immune cells [5]. Transcript-specific expression may be regulated in a cell type- or condition-specific manner [2, 3].
In this study, $\mathrm{CD}^{+}$and $\mathrm{CD}^{+}{ }^{+} \mathrm{T}$-cell ASE appear largely similar, but the degree of correlation with other immune cells is unclear. Hence, investigation of a more diverse cell type range is warranted.

Transcript-specific expression may provide a more robust molecular signature than overall gene expression as biological classifier [2]. Hence, transcript-specific regulation by disease associated variants, as highlighted for MS in this study, is an emerging genetic concept with potential translational implications.

Acknowledgements $\mathrm{LVH}$ holds a $\mathrm{PhD}$ Fellowship of the Belgian Charcot Foundation. AG is supported for work related to this topic by the Research Council KU Leuven, the Research Foundation Flanders and the Queen Elisabeth Medical Foundation.

\section{Compliance with ethical standards}

Conflict of interest The authors declare that they have no conflict of interest.

Publisher's note Springer Nature remains neutral with regard to jurisdictional claims in published maps and institutional affiliations.

\section{References}

1. Ban M, Liao W, Baker A, Thorpe J, Molyneux P, Fraser M, et al. Transcript specific regulation of expression influences susceptibility to multiple sclerosis. Eur J Hum Genet. 2020. in press.

2. Park E, Pan Z, Zhang Z, Lin L, Xing Y. The expanding landscape of alternative splicing variation in human populations. Am J Hum Genet. 2018;102:11-26.

3. Li YI, van de Geijn B, Raj A, Knowles DA, Petti AA, Golan D, et al. RNA splicing is a primary link between genetic variation and disease. Science. 2016;352:600-4.

4. Chun S, Casparino A, Patsopoulos NA, Croteau-Chonka DC, Raby BA, De Jager PL, et al. Limited statistical evidence for shared genetic effects of eQTLs and autoimmune-disease-associated loci in three major immune-cell types. Nat Genet. 2017;49:600-5.

5. The International Multiple Sclerosis Genetics Consortium. Multiple sclerosis genomic map implicates peripheral immune cells and microglia in susceptibility. Science. 2019;365:eaav7188. 